\title{
Provisional drug-coated balloon treatment guided by physiology on de novo coronary lesion
}

\author{
Eun-Seok Shin ${ }^{1}$, Liew Houng Bang ${ }^{2}$, Eun Jung Jun ${ }^{1}$, Ae-Young Her ${ }^{3}$, \\ Ju-Hyun Chung ${ }^{1}$, Scot Garg ${ }^{4}$, Joo Myung Lee ${ }^{5}$, Joon-Hyung Doh ${ }^{6}$, \\ Chang-Wook Nam ${ }^{7}$, Bon-Kwon Koo ${ }^{8}$, Qiang Tang ${ }^{9}$ \\ ${ }^{1}$ Division of Cardiology, Ulsan Medical Center, Ulsan Hospital, Ulsan, South Korea \\ ${ }^{2}$ Cardiology Department and Clinical Research Center, Queen Elizabeth Hospital II, Kota, Kinabalu, Malaysia \\ ${ }^{3}$ Department of Internal Medicine, Kangwon National University School of Medicine, Chuncheon, South Korea \\ ${ }^{4}$ East Lancashire Hospitals NHS Trust, Blackburn, Lancashire, United Kingdom \\ ${ }^{5}$ Department of Internal Medicine and Cardiovascular Center, Heart Vascular Stroke Institute, \\ Samsung Medical Center, Sungkyunkwan University School of Medicine, Seoul, South Korea \\ ${ }^{6}$ Division of Cardiology, Inje University Ilsan Paik Hospital, Goyang, South Korea \\ ${ }^{7}$ Department of Internal Medicine, Keimyung University Dongsan Medical Center, Daegu, South Korea \\ ${ }^{8}$ Department of Internal Medicine and Cardiovascular Center, \\ Seoul National University Hospital, Seoul, South Korea \\ ${ }^{9}$ Division of Cardiology, Department of Internal Medicine, Beijing University Shougang Hospital, Beijing, China
}

\begin{abstract}
Although drug-eluting stents (DES) have become the mainstay of percutaneous coronary intervention, late and very late stent thrombosis remains a concern. Drug-coated balloons (DCB) have the advantage of preserving the anti-restenotic benefits of DES while minimizing potential long-term safety concerns. Currently the two methods to ensure successful DCB treatment of a stenotic lesion are angiography or physiology-guided DCB application. This review will evaluate these two methods based on previous evidence and make suggestions on how to perform DCB treatment more efficiently and safely. (Cardiol J 2021; 28, 4: 615-622)
\end{abstract}

Key words: drug-coated balloon, coronary artery disease, physiology, fractional flow reserve, diameter stenosis, de novo lesion

\section{Introduction}

The successful restoration of coronary flow is the goal of percutaneous coronary intervention (PCI) for obstructive coronary artery disease. Due to the limitations of accurately measuring blood flow in clinical practice, anatomical assessment using diameter stenosis (DS) or minimal lumen diameter by coronary angiography has been used to guide the procedure. However, coronary angiography alone is inherently limited by its inability to provide information pertaining to the functional significance of stenoses. Fractional flow reserve (FFR) was developed as a technique to enable physiological assessment of coronary lesions. FFR expresses the maximum achievable blood flow to the myocardium supplied by a stenotic artery as a fraction of normal maximum flow. As such, it provides an objective measure of the hemodynamic significance of an epicardial stenosis and FFR-guided PCI is associated with a better prognosis than angiography-guided PCI [1].

Drug-coated balloons (DCB) provide local drug delivery after successful balloon angioplasty

Address for correspondence: Eun-Seok Shin, MD, PhD, 13, Wolpyeong-ro, 171beon-gil, Nam-gu, Ulsan, 44686,

Republic of Korea, tel: +82-52-259-5425, fax: +82-52-259-5117, e-mail: sesim1989@gmail.com

Received: 3.04.2020

Accepted: 25.07.2020

This article is available in open access under Creative Common Attribution-Non-Commercial-No Derivatives 4.0 International (CC BY-NC-ND 4.0) license, allowing to download articles and share them with others as long as they credit the authors and the publisher, but without permission to change them in any way or use them commercially. 
(BA) to inhibit restenosis, however concerns over acute vessel closure, have hampered their use for de novo lesions. During the BA era, Bech et al. [2] demonstrated that patients with a residual DS of $\leq 35 \%$ and an FFR value after BA of $\geq 0.90$ had excellent clinical outcomes to 2 -years. Further, in a previous study, it was demonstrated that DCB treatment after successful BA with a resultant FFR value $\geq 0.75$ was safe and effective, without the additional risk of acute vessel closure [3].

One of the advantages using a DCB over drug-eluting stent (DES) is the short duration of dual antiplatelet therapy (DAPT) especially in patients with high risk of bleeding, and in those with contraindications to long term DAPT, since they require only 1 month of DAPT. Although the latest data from the Onyx ONE and EVOVE Short DAPT studies demonstrated that 1 to 3 months of DAPT is noninferior to the standard 12 -month DAPT in terms of the risk of stent thrombosis $[4,5]$, neoatherosclerosis with DES is inevitable over time, and this is known to progress faster than de novo lesions, resulting in stent failure manifesting as restenosis and thrombosis [6]. Nevertheless, unlike the BA era, there are now two available options - DCB and DES - for the local delivery of antiproliferative drugs allowing patients to receive different treatment options. There have been many efforts to examine the pros and cons of DCB compared to DES, and an indication standard for DCB treatment is expected in the near future. This review discusses a safe and effective method for the use of DCBs in the treatment of obstructive coronary artery disease.

\section{Optimal lesion preparation for DCB application}

The use of DCBs has been proven to be very effective for in-stent restenosis (ISR) and is recommended by European, German, and Asia-Pacific consensus groups [7-9]. Although DCB treatment is a reasonable option for ISR, recurrent target lesion failure (TLF) still occurs in some patients after treatment. Optimal lesion preparation for ISR plays an important role in reducing adverse clinical outcomes after DCB treatment. The RIBS IV study investigated the treatment of patients with DES-ISR, and showed that re-stenting with a DES reduced the 1-year composite outcome of cardiac death, myocardial infarction (MI), and target vessel revascularization (TVR) compared with using a DCB (10\% vs. $18 \%$; hazard ratio [HR]: 0.58; 95\% confidence interval [CI]: 0.35-0.98; $\mathrm{p}=0.04)$. However, this benefit was driven in part due to sub-optimal lesion preparation and inadequate flow after balloon angioplasty in the DCB arm [10]. Another recent study showed that modifiable independent predictors of recurrent TLF were residual DS after lesion preparation, DCB-to-stent ratio, and DCB inflation time [11]. In their study, TLF occurred in $20.3 \%$ and the best cutoff values were $20 \%, 0.91$, and $60 \mathrm{~s}$ for residual DS, DCB-to-stent ratio, and DCB inflation time, respectively. TLF rates were significantly higher in groups with residual $\mathrm{DS} \geq 20 \%$ (34.7\% vs. $12.5 \%$; HR: 2.15; 95\% CI: 1.86-2.48; p < 0.001), DCB-tostent ratio $\leq 0.91$ ( $46.4 \%$ vs. $21.9 \%$; HR: $2.02 ; 95 \%$ CI: $1.75-2.34 ; \mathrm{p}<0.001)$, and inflation time $\leq 60 \mathrm{~s}$ (26.2\% vs. $14.0 \%$; HR: 1.82 ; 95\% CI: $1.36-2.45$; $\mathrm{p}<0.001)$. When classifying ISR lesions by combination of three procedure-related factors, TLF occurred in $8.3 \%$ in the fully optimized procedure group and $66.7 \%$ in the non-optimized group $(\mathrm{p}<0.001)$, demonstrating clearly that fully optimized DCB treatment with ideal lesion preparation, sufficient dilatation, and prolonged inflation could reduce TLF. Appropriate lesion preparation can create an environment that allows homogeneous drug delivery to the lesion efficiently and thus, has a significant impact on the efficacy and safety of DCB treatment.

There is no evidence of criteria for optimal lesion preparation for de novo coronary artery lesions. In ISR as well as in de novo lesions, conventional lesion preparation is performed using a non- or semi-compliant balloon. In complex lesions, however, the use of high-pressure noncompliant balloons or scoring/cutting balloons should be considered to provide better lesion preparation. The shortcomings of conventional balloon angioplasty include balloon slippage and edge dissections, post procedure, and these problems could be reduced with the use of a scoring balloon, which may also allow enhanced local drug uptake.

In an early intravascular ultrasound evaluation study comparing cutting balloons with conventional balloons for the treatment of ISR, the luminal area acute gain was larger in the cutting balloon group due to more effective tissue extrusion, while late loss was smaller [12]. Cutting or scoring the neointimal plaque lessens the elastic and fibrotic continuity of the internal fibrous layer and makes the tissue more amenable to being pushed outward through the stent struts. In the ISAR-DESIRE 4 study, neointimal modification with scoring balloon before DCB was compared with standard DCB therapy in patients with ISR [13]. Pre-dilatation with a scoring balloon resulted in a significantly lower 
rate of in-segment percentage DS $(35.0 \pm 16.8 \%$ vs. $40.4 \pm 21.4 \%, \mathrm{p}=0.047)$ and binary angiographic restenosis rate $(18.5 \%$ vs. $32.0 \%, \mathrm{p}=0.026)$ at 6-8 month follow-up. The results demonstrated that the use of a scoring balloon improves the complete expansion of a re-stenosed stent, neointimal modification, and homogeneous drug delivery and hence, increases the anti-restenotic efficacy of DCB treatment.

ST-segment elevation myocardial infarction (STEMI) is the most representative disease of atherothrombotic lesions. In STEMI patients, stent implantation reduces target lesion revascularization (TLR), however, it tends to increase the long-term risk of stent-related events such as stent thrombosis and ISR [14-16]. In the majority of STEMI patients, rapid restoration of coronary flow is the main purpose of treatment, and this can be achieved by a combined approach of pharmacologic and interventional treatments without stenting. Thrombus aspiration is an adjunctive non-pharmacological strategy during primary PCI designed to improve epicardial and myocardial reperfusion. However, recent studies failed to show the clinical benefit of aspiration thrombectomy in STEMI patients due to insufficient removal of thrombus at the culprit lesion [17-19].

Stenting has been shown to reduce the need for TVR in acute MI, however, this was not associated with a significant reduction in mortality or reinfarction compared with BA [20]. To avoid the long-term risk of stent-related events in STEMI patients, a DCB strategy in primary PCI could be a safe and feasible alternative strategy to DES treatment if coronary flow is restored and no significant residual stenosis persists after balloon dilatation or thrombo-suction. Before using a DCB, successful thrombus aspiration is important and has beneficial effects considering that adequate lesion preparation helps facilitate homogeneous drug delivery. In the REVELATION study, a DCB strategy was noninferior to DES in STEMI patients in terms of FFR assessed at 9 months $(0.92 \pm 0.05$ vs. $0.91 \pm$ $\pm 0.06, \mathrm{p}=0.27$ ), and during follow-up, only $2 \mathrm{pa}-$ tients received a non-urgent TLR ( 1 in each group) [21]. One option in STEMI patients where coronary flow is restored and no significant residual stenosis is observed after balloon pre-dilatation, is medical treatment with an anticoagulant and antiplatelet agent without any further immediate intervention, followed by a repeat coronary angiogram after 1-2 weeks to decide whether to use a DCB or DES. The high risk of restenosis and stent thrombosis in patients with a chronic total occlusion (CTO) is still a major problem. In the PEPCAD-CTO study, the use of DCB plus BMS was associated with similar clinical results and a non-significantly higher in-stent late loss compared with DES [22]. The DCB only approach studies for CTO cases are scarce but recent registry data has suggested it was a feasible and well-tolerated treatment method if the pre-dilatation result is good [23]. In their feasibility and safety study, the incidence of angiographic restenosis was $11.8 \%$ at mean 8 -month follow-up, which were similar or lower than prior CTO studies using either DES or BMS. Furthermore, late lumen gain was found in $67.6 \%$ of patients and was caused by an increase in vessel size rather than plaque regression.

When a DES is used after successful recanalization of a CTO, the stent may be undersized because the lesion was occluded, and the vessel did not grow soon after reperfusion. CTO lesions have negative remodeled distal vessels because they have not had any flow for a long time. After $\mathrm{BA}$, antegrade flow increases and vessels become larger, however this may take from several weeks to months. Therefore, immediately after balloon angioplasty of a CTO, it is easy to under-estimate the true vessel size, increasing the risk of stent under-sizing and subsequent risks of restenosis, late stent mal-apposition and stent thrombosis. Moreover, the metallic cage can inhibit positive remodeling leaving a small luminal size after vessel recovery. However, after treatment with a DCB, it is possible that vessels will return to their original size over time, which is one of the greatest advantages of DCB treatment in CTO lesions. Furthermore, in cases of DCB only treatment, the presence of heavy calcification could support the vessel as a DES does, and could give the vessel a chance to grow and heal. However, larger, randomized controlled trials are necessary to further evaluate the DCB-only approach for CTO lesions.

\section{Provisional strategy guided by physiology}

According to the German Consensus Group, lesion characteristics determined by angiography after BA can identify acceptable lesion preparation by assessing for the absence of a flow-limiting dissection and a non-significant residual DS $\leq 30 \%$ [8]. Angiographically significant parameters after BA are residual DS $>30 \%$ or dissection type $\mathrm{C}$ or more. Recently it was demonstrated that DCB treatment could be performed safely and effectively after successful BA (Thrombolysis In Myocardial Infarction [TIMI] flow grade 3 after BA) with an FFR value $\geq 0.75$, without any increased risk of 


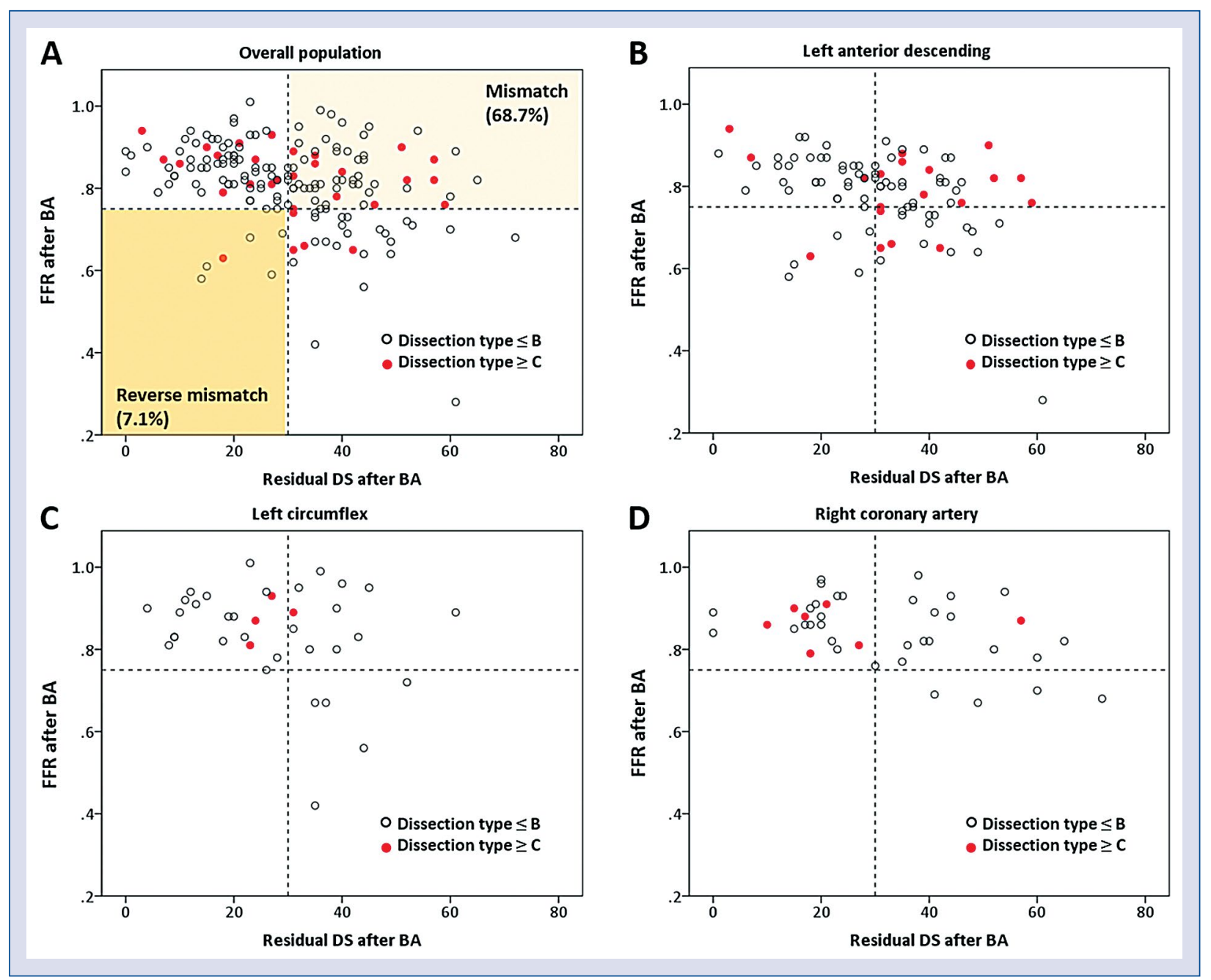

Figure 1. Correlation between fractional flow reserve (FFR) after balloon angioplasty (BA) and residual diameter stenosis (DS); A. Overall population; B. Left anterior descending artery; C. Left circumflex artery; D. Right coronary artery. Reused with permission from: [1].

acute vessel closure and a lower rate of restenosis. Of note, a high frequency of non-concordance was found between angiographic and functional characteristics when using an FFR after BA cutoff of 0.75 to define functionally significant lesions [1]. The results showed a mismatch of $68.7 \%$ (residual $\mathrm{DS}>30 \%$ and FFR afterBA $\geq 0.75$ ) with areverse mismatch (residual DS $\leq 30 \%$ and FFR after $\mathrm{BA}<0.75$ ) in $7.1 \%$ (Fig. 1A). If these mismatch lesions were treated solely using angiography, as recommended by the German consensus group, all of them should be treated with stent implantation, since the residual DS was above 30\%, even though the post-balloon FFR was $>0.75$. Previous mid- and long-term follow-up studies in patients with an FFR after $\mathrm{BA} \geq 0.75$ showed comparable clinical outcomes between DCB and stent treatments $[1,3,24]$. Therefore, FFR-guided DCB treatment could safely reduce the number of unnecessary stent implantations in this mismatch population. In the reverse mismatch population, the guidelines suggest using a DCB over a stent, since the residual DS was $\leq 30 \%$. If the guidelines are followed, only severe dissections (type $\mathrm{C}$ dissection) should be treated with a stent, whilst the rest of them could receive DCB treatment $(6.0 \%$ of DS $\leq 30 \%$ ) [1]. It is well known that in patients with functionally significant stenoses, FFR-guided PCI decreases the need of urgent revascularization compared with medical therapy alone [25]. Thus, these reverse mismatch lesions pose a higher clinical risk of future events and it is appropriate for them to be treated with stent implantation.

In the left anterior descending artery (LAD) the majority of lesions with severe dissection had an FFR after BA of $\geq 0.75$ which was independent 


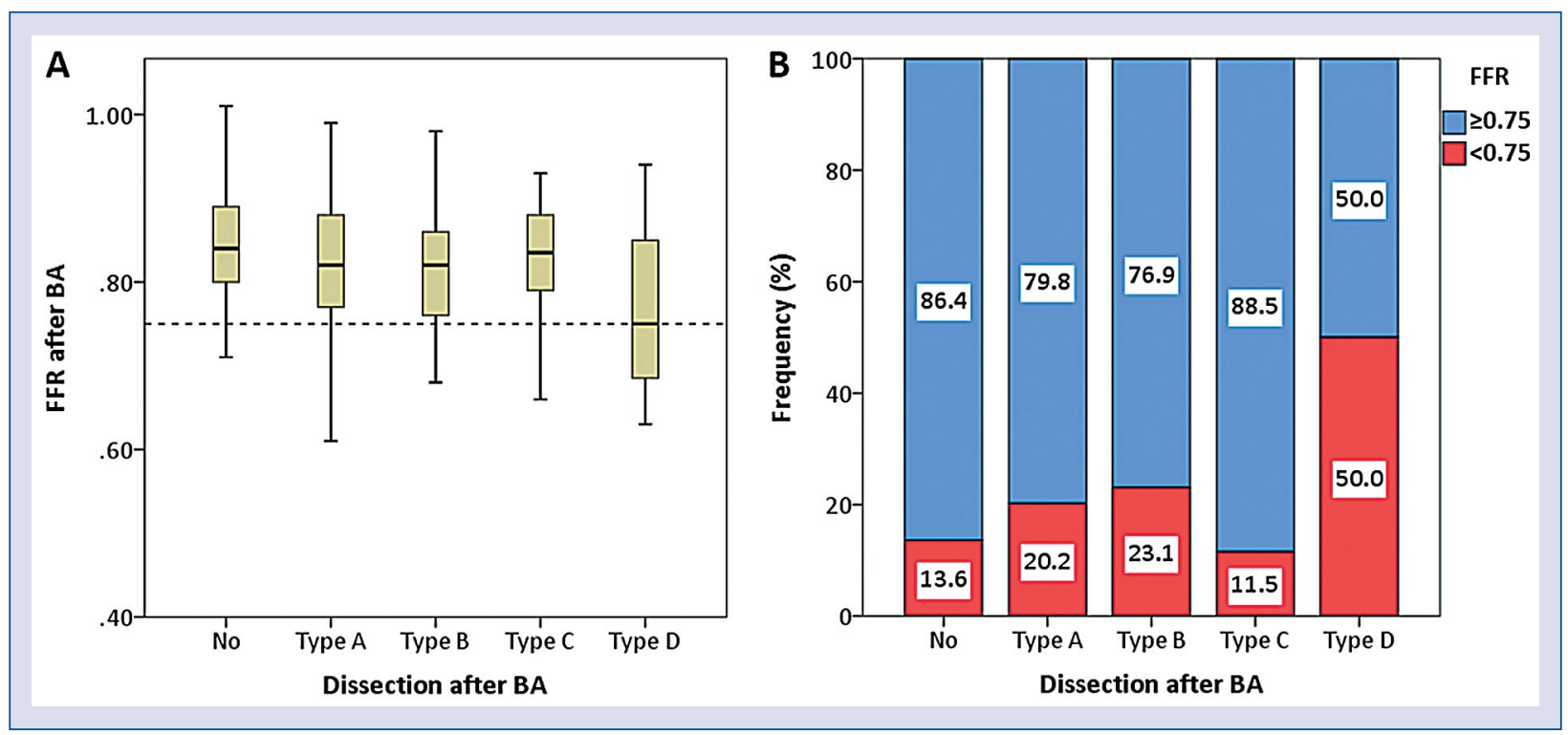

Figure 2. Fractional flow reserve (FFR) after balloon angioplasty (BA) according to dissection type; A. Distribution of FFR; B. Proportion of high and low FFR groups. Modified with permission from: [1].

of the severity of the dissection (Fig. 1B). Of note, all severe dissections in non-LAD lesions had an FFR after $\mathrm{BA} \geq 0.75$ (Fig. 1C, D). The dissection type after BA does not correlate with residual FFR as seen in Figure 2. Thus, FFR after BA measurements in LAD lesions could be recommended not just to reduce the number of stents in mismatch lesions, but also to prevent future adverse clinical events in reverse mismatch lesions, while lesions in the circumflex and right coronary artery could be safely treated with angiography alone. FFR-guided DCB treatment has several advantages compared to angiography-guided DCB application. A recent recommendation from an Asia-Pacific consensus group reported both angiographic and functional criteria for large de novo coronary lesions [9].

The provisional strategy guided by physiology for de novo lesions are summarized in Figure 3. The first step for successful DCB treatment is to achieve optimal lesion preparation by pre-dilation balloon (non-compliant or scoring/cutting balloons) or a non-balloon device like atherectomy or rotablator. A balloon to artery ratio of 0.8 to 1.0 and an inflation pressure higher than nominal should be used. Thrombus aspiration for patients with STEMI should be performed in appropriate situations. The acceptable angiographic and functional criteria after BA to perform DCB treatment are TIMI grade 3 flow and FFR $\geq 0.75$. In this FFR-guided DCB strategy, if the FFR after $\mathrm{BA}$ is $\geq 0.75$, DCB treatment can be performed

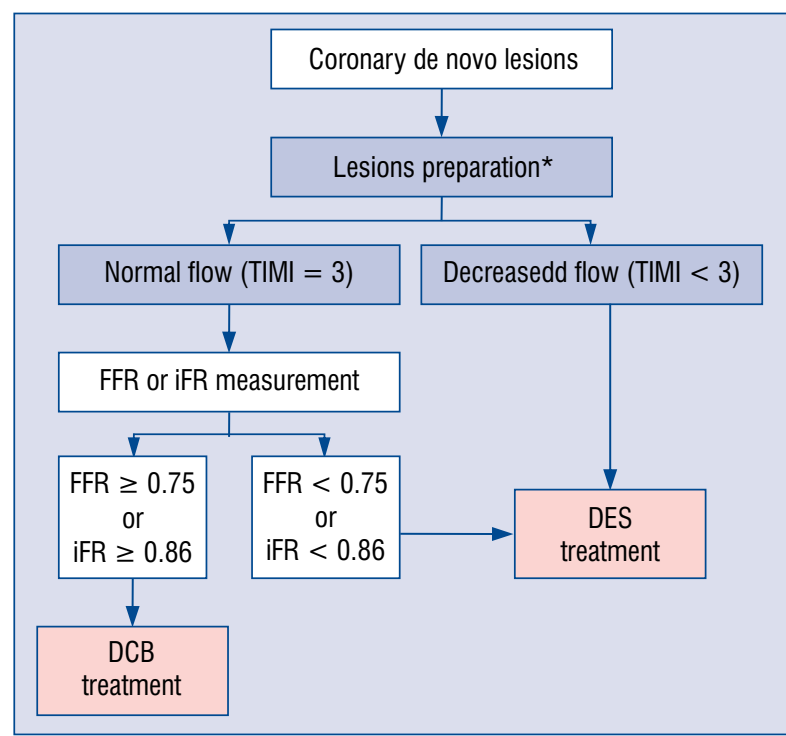

Figure 3. Provisional drug-coated balloon (DCB) strategy guided by fractional flow reserve (FFR). The acceptable angiographic and functional criteria after balloon angioplasty to perform DCB treatment are Thrombolysis In Myocardial Infarction (TIMI) 3 grade flow and FFR $\geq 0.75$; iFR - instantaneous wave-free ratio; DES drug-eluting stent. *Lesion preparation by optimal sized balloon (balloon-to-vessel ratio $0.8-1.0$ ) or non-balloon catheters.

safely [3, 24]. The provisional DCB treatment guided by physiology aims to provide safer DCB treatment based on physiology according to all 


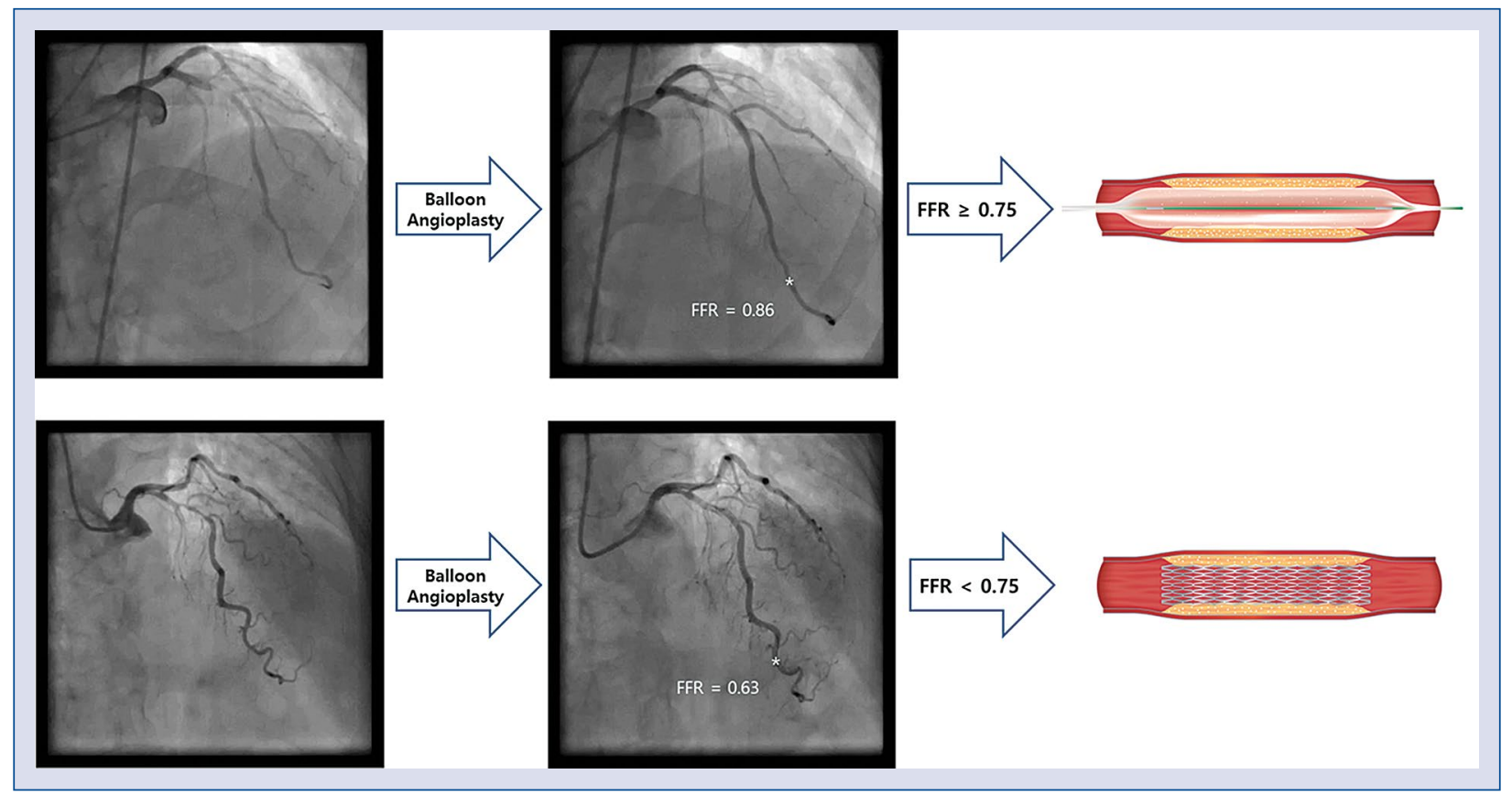

Figure 4. Representative cases for provisional drug-coated balloon strategy guided by fractional flow reserve (FFR).

anatomical changes like dissection presence or severity, and residual stenosis occurring after BA. In a previous study comparing angiography to FFR-guided DCB treatment for 167 lesions, there was a mismatch in $68.7 \%$ (57 of 83 ) of the population and a reverse mismatch in $7.1 \%$ (6 of 84) [1]. If an angiography-guided strategy was used, $57.5 \%$ (96 of 167) would need treatment with a DES, whilst using an FFR-guided strategy only 19.2\% (32 of 167) need a DES; this $66.7 \%$ reduction in stent usage occurred without increasing safety concerns. Lesions in the reverse mismatch population pose a higher clinical risk of future events however whilst the angio-guided approach recommends treating with a DCB since the residual DS is $<30 \%$, the FFR-guided approach recommends treating with a DES since the residual FFR is $<0.75$ and hence, it could prevent possible future events.

Recently, it was demonstrated that instantaneous wave-free ratio (iFR), an alternative measure that does not require the administration of adenosine, measured right after BA is safe and effective for de novo coronary lesions [26]. The cutoff value of iFR right after BA used to define functionally nonsignificant residual stenotic lesions was 0.86 that iFR-guided paclitaxel-coated balloon treatment is safe and effective for de novo major epicardial coronary lesions with good anatomical patency at 9-month follow-up and showed good long-term clinical outcomes in patients with iFR $\geq 0.86$ after BA. As alternative methods to FFR or iFR, functional coronary imaging, quantitative flow ratio, have recently emerged, allowing wire-free functional assessment of stenosis severity based on a computational fluid dynamics model or mathematical assumptions of coronary flow. Although previous studies have demonstrated excellent correlations and diagnostic agreements with FFR [27, 28], an evaluation of the diagnostic performance and agreement of QFR using FFR or iFR as reference standards in the situation of BA is needed (Figs. 3, 4).

\section{Medical treatment}

Easy to heal and the lower risk of target lesion thrombosis in lesions treated with a DCB makes prolonged DAPT therapy unnecessary. The European Society of Cardiology guideline recommends 6 months of DAPT treatment, however, shorter durations of 1-3 months after DCB have not been associated with any increased risk of long-term adverse outcomes compared with BMS or DES [7]. Looking at the previous data, when $1^{\text {st }}$ generation DES was used, stent thrombosis occurred $0.6 \%$ per year [29], and $2^{\text {nd }}$ generation stent occurred $0.3 \%$ of stent thrombosis per year [30]. In the BASKET--SMALL 2 study for small de novo lesions 
( $\mathrm{n}=758$ patients), DCB was non-inferior to DES for major adverse cardiovascular events (cardiac death, non-fatal MI, and target vessel revascularization) for up to 12 months $(7.5 \%$ vs. $7.3 \%$; HR 0.97 [95\% CI 0.58-1.64], $p=0.918$ ) [31]. The thrombosis rate (probable or definite) in DCB treated lesions was numerically lower than DES (0.8\% vs. $1.1 \%$; HR 0.73 [0.16-3.26]) despite DAPT being continued for only 1 month in the DCB arm and 6-12 months in the DES arm. Major bleeding in DCB arm was also numerically lower than the DES arm (1.1\% vs. $2.4 \%$; HR 0.45 [0.14-1.46]).

High-intensity statin therapy may provide incremental clinical benefits after DCB application. It is well known that statin treatment causes plaque regression and improves clinical outcomes when used for either primary or secondary prevention. BA creates iatrogenic plaque dissection and causes plaque redistribution, and $\mathrm{DCB}$ provides local drug delivery to prevent restenosis. After DCB treatment, cholesterol-lowering drugs may cause greater plaque regression compared to statin only treatment without PCI. Regarding the effects of statin therapy, a previous study demonstrated that a clear reduction of lipid core was only observed in thin-cap fibroatheromas, suggesting that changes in plaque composition following statin therapy might occur earlier and to a greater degree in vulnerable plaque compared to stable plaque [32]. Another study showed that DCB treatment with high dose statins caused persistent patency with plaque redistribution without chronic elastic recoil and restored coronary blood flow resulting in increased lumen areas at follow-up [33]. These results suggest that there will be regression of plaque after DCB treatment through high dose statin therapy. Therefore, high intensity statin therapy can reinforce the efficacy of DCB treatment.

\section{Conclusions}

For successful DCB treatment, optimal lesion preparation using optimally sized scoring balloons is essential. Ideal lesion preparation should be assessed by measuring flow status using physiological indexes such as FFR rather than by estimating stenotic severity from angiography alone. Although not all coronary lesions require FFR measurement, if the lesion subtends a large amount of myocardium such as lesions in the proximal LAD, they should be assessed with FFR. The physiology-guided provisional strategy suggests that DCB treatment should only be performed if adequate coronary flow is obtained after optimal balloon angioplasty, with newer generation DES used in cases of inadequate flow. For successful DCB treatment, there is a need for better technology that can make plaque modifications safe and effective. In addition, the importance of medical treatment to maximize the effect of DCB (anti-thrombotics and cholesterollowering drugs) cannot be overemphasized.

Conflict of interest: Dr. Bon-Kwon Koo has received institutional research grant from St. Jude Medical. Dr. Joo Myung Lee received a research grant from St. Jude Medical (Abbott Vascular) and Philips Volcano. All other authors declare that there is no conflict of interest relevant to the submitted work.

\section{References}

1. Chung JH, Lee KE, Her AY, et al. Comparison of fractional flow reserve and angiographic characteristics after balloon angioplasty in de novo coronary lesions. Int J Cardiovasc Imaging. 2019; 35(11): 1945-1954, doi: 10.1007/s10554-019-01649-y, indexed in Pubmed: 31214851.

2. Bech GJ, Pijls NH, De Bruyne B, et al. Usefulness of fractional flow reserve to predict clinical outcome after balloon angioplasty. Circulation. 1999; 99(7): 883-888, doi: 10.1161/01.cir.99.7.883, indexed in Pubmed: 10027810.

3. Her AY, Shin ES, Lee JM, et al. Paclitaxel-coated balloon treatment for functionally nonsignificant residual coronary lesions after balloon angioplasty. Int J Cardiovasc Imaging. 2018; 34(9): 1339-1347, doi: 10.1007/s10554-018-1351-z, indexed in Pubmed: 29696453.

4. Kedhi E, Latib A, Abizaid A, et al. Rationale and design of the Onyx ONE global randomized trial: A randomized controlled trial of high-bleeding risk patients after stent placement with 1 month of dual antiplatelet therapy. Am Heart J. 2019; 214: 134-141, doi: 10.1016/j.ahj.2019.04.017, indexed in Pubmed: 31203158.

5. Mauri L, Kirtane AJ, Windecker S, et al. Rationale and design of the EVOLVE Short DAPT Study to assess 3-month dual antiplatelet therapy in subjects at high risk for bleeding undergoing percutaneous coronary intervention. Am Heart J. 2018; 205: 110-117, doi: 10.1016/j.ahj.2018.08.004, indexed in Pubmed: 30218844.

6. Yahagi K, Kolodgie FD, Otsuka F, et al. Pathophysiology of native coronary, vein graft, and in-stent atherosclerosis. Nat Rev Cardiol. 2016; 13(2): 79-98, doi: 10.1038/nrcardio.2015.164, indexed in Pubmed: 26503410.

7. Neumann FJ, Sousa-Uva M, Ahlsson A, et al. 2018 ESC/EACTS Guidelines on myocardial revascularization. Eur Heart J. 2019; 40(2): 87-165, doi: 10.1093/eurheartj/ehy394, indexed in Pubmed: 30165437.

8. Kleber FX, Rittger H, Bonaventura K, et al. Drug-coated balloons for treatment of coronary artery disease: updated recommendations from a consensus group. Clin Res Cardiol. 2013; 102(11): 785-797, doi: 10.1007/s00392-013-0609-7, indexed in Pubmed: 23982467.

9. Her AY, Shin ES, Bang LH, et al. Drug-coated balloon treatment in coronary artery disease: Recommendations from an AsiaPacific Consensus Group. Cardiol J. 2021; 28(1): 136-149, doi: 10.5603/CJ.a2019.0093, indexed in Pubmed: 31565793. 
10. Alfonso F, Pérez-Vizcayno MJ, Cárdenas A, et al. A Prospective Randomized Trial of Drug-Eluting Balloons Versus EverolimusEluting Stents in Patients With In-Stent Restenosis of DrugEluting Stents: The RIBS IV Randomized Clinical Trial. J Am Coll Cardiol. 2015; 66(1): 23-33, doi: 10.1016/j.jacc.2015.04.063, indexed in Pubmed: 26139054.

11. Rhee TM, Lee JM, Shin ES, et al. Impact of optimized procedurerelated factors in drug-eluting balloon angioplasty for treatment of in-stent restenosis. JACC Cardiovasc Interv. 2018; 11(10): 969-978, doi: 10.1016/j.jcin.2018.02.002, indexed in Pubmed: 29798774.

12. Muramatsu T, Tsukahara R, Ho M, et al. Efficacy of cutting balloon angioplasty for in-stent restenosis: an intravascular ultrasound evaluation. J Invasive Cardiol. 2001; 13(6): 439-444, indexed in Pubmed: 11385165.

13. Kufner S, Joner M, Schneider S, et al. ISAR-DESIRE 4 Investigators. Neointimal Modification With Scoring Balloon and Efficacy of Drug-Coated Balloon Therapy in Patients With Restenosis in Drug-Eluting Coronary Stents: A Randomized Controlled Trial. JACC Cardiovasc Interv. 2017; 10(13): 1332-1340, doi: 10.1016/j. jcin.2017.04.024, indexed in Pubmed: 28683939.

14. Gonzalo N, Barlis P, Serruys PW, et al. Incomplete stent apposition and delayed tissue coverage are more frequent in drugeluting stents implanted during primary percutaneous coronary intervention for ST-segment elevation myocardial infarction than in drug-eluting stents implanted for stable/unstable angina: insights from optical coherence tomography. JACC Cardiovasc Interv. 2009; 2(5): 445-452, doi: 10.1016/j.jcin.2009.01.012, indexed in Pubmed: 19463469.

15. Nakazawa G, Finn AV, Joner M, et al. Delayed arterial healing and increased late stent thrombosis at culprit sites after drug-eluting stent placement for acute myocardial infarction patients: an autopsy study. Circulation. 2008; 118(11): 1138-1145, doi: 10.1161/ CIRCULATIONAHA.107.762047, indexed in Pubmed: 18725485.

16. Stone SG, Serrao GW, Mehran R, et al. Incidence, predictors, and implications of reinfarction after primary percutaneous coronary intervention in ST-segment-elevation myocardial infarction: the Harmonizing Outcomes with Revascularization and Stents in Acute Myocardial Infarction Trial. Circ Cardiovasc Interv. 2014; 7(4): 543-551, doi: 10.1161/CIRCINTERVENTIONS.114.001360, indexed in Pubmed: 24939928.

17. Fröbert O, Lagerqvist Bo, Olivecrona GK, et al. TASTE Trial. Thrombus aspiration during ST-segment elevation myocardial infarction. N Engl J Med. 2013; 369(17): 1587-1597, doi: 10.1056/ NEJMoa1308789, indexed in Pubmed: 23991656.

18. Higuma T, Soeda T, Yamada M, et al. Does residual thrombus after aspiration thrombectomy affect the outcome of primary PCI in patients with ST-segment elevation myocardial infarction? An optical coherence tomography study. JACC Cardiovasc Interv. 2016; 9(19): 2002-2011, doi: 10.1016/j.jcin.2016.06.050, indexed in Pubmed: 27712735.

19. Jolly SS, Cairns JA, Yusuf S, et al. TOTAL Investigators. Randomized trial of primary PCI with or without routine manual thrombectomy. N Engl J Med. 2015; 372(15): 1389-1398, doi: 10.1056/NEJMoa1415098, indexed in Pubmed: 25853743.

20. De Luca G, Suryapranata H, Stone GW, et al. Coronary stenting versus balloon angioplasty for acute myocardial infarction: a meta-regression analysis of randomized trials. Int J Cardiol. 2008; 126(1): 37-44, doi: 10.1016/j.ijcard.2007.03.112, indexed in Pubmed: 17544528.

21. Vos NS, Fagel ND, Amoroso G, et al. Paclitaxel-Coated balloon angioplasty versus drug-eluting stent in acute myocardial in- farction: the REVELATION randomized trial. JACC Cardiovasc Interv. 2019; 12(17): 1691-1699, doi: 10.1016/j.jcin.2019.04.016, indexed in Pubmed: 31126887.

22. Wöhrle J, Werner GS. Paclitaxel-coated balloon with bare-metal stenting in patients with chronic total occlusions in native coronary arteries. Catheter Cardiovasc Interv. 2013; 81(5): 793-799, doi: 10.1002/ccd.24409, indexed in Pubmed: 22511572.

23. Köln PJ, Scheller B, Liew HB, et al. Treatment of chronic total occlusions in native coronary arteries by drug-coated balloons without stenting - A feasibility and safety study. Int J Cardiol. 2016; 225: 262-267, doi: 10.1016/j.ijcard.2016.09.105, indexed in Pubmed: 27741486.

24. Shin ES, Ann SH, Balbir Singh G, et al. Fractional flow reserveguided paclitaxel-coated balloon treatment for de novo coronary lesions. Catheter Cardiovasc Interv. 2016; 88(2): 193-200, doi: 10.1002/ccd.26257, indexed in Pubmed: 26423017.

25. De Bruyne B, Pijls NHJ, Kalesan B, et al. FAME 2 Trial Investigators. Fractional flow reserve-guided PCI versus medical therapy in stable coronary disease. N Engl J Med. 2012; 367(11): 991-1001, doi: 10.1056/NEJMoa1205361, indexed in Pubmed: 22924638.

26. Chung JH, Shin ES, Her AY, et al. Instantaneous wave-free ratioguided paclitaxel-coated balloon treatment for de novo coronary lesions. Int J Cardiovasc Imaging. 2020; 36(2): 179-185, doi: 10.1007/s10554-019-01707-5, indexed in Pubmed: 31598811.

27. Collet C, Onuma Y, Sonck J, et al. Diagnostic performance of angiography-derived fractional flow reserve: a systematic review and Bayesian meta-analysis. Eur Heart J. 2018; 39(35): 3314-3321, doi: 10.1093/eurheartj/ehy445, indexed in Pubmed: 30137305.

28. Xu Bo, Tu S, Qiao S, et al. Diagnostic accuracy of angiographybased quantitative flow ratio measurements for online assessment of coronary stenosis. J Am Coll Cardiol. 2017; 70(25): 3077-3087, doi: 10.1016/j.jacc.2017.10.035, indexed in Pubmed: 29101020.

29. Daemen J, Wenaweser P, Tsuchida K, et al. Early and late coronary stent thrombosis of sirolimus-eluting and paclitaxel-eluting stents in routine clinical practice: data from a large two-institutional cohort study. Lancet. 2007; 369(9562): 667-678, doi: 10.1016/S0140-6736(07)60314-6, indexed in Pubmed: 17321312.

30. von Birgelen C, van der Heijden LC, Basalus MWZ, et al. FiveYear outcome after implantation of zotarolimus- and everolimuseluting stents in randomized trial participants and nonenrolled eligible patients: a secondary analysis of a randomized clinical trial. JAMA Cardiol. 2017; 2(3): 268-276, doi: 10.1001/jamacardio.2016.5190, indexed in Pubmed: 28114618.

31. Jeger RV, Farah A, Ohlow MA, et al. BASKET-SMALL 2 Investigators. Drug-coated balloons for small coronary artery disease (BASKET-SMALL 2): an open-label randomised non-inferiority trial. Lancet. 2018; 392(10150): 849-856, doi: 10.1016/S01406736(18)31719-7, indexed in Pubmed: 30170854.

32. Hwang DS, Shin ES, Kim SJ, et al. Early differential changes in coronary plaque composition according to plaque stability following statin initiation in acute coronary syndrome: classification and analysis by intravascular ultrasound-virtual histology. Yonsei Med J. 2013; 54(2): 336-344, doi: 10.3349/ymj.2013.54.2.336, indexed in Pubmed: 23364965.

33. Ann SH, Balbir Singh G, Lim KH, et al. Anatomical and Physiological Changes after Paclitaxel-Coated Balloon for Atherosclerotic De Novo Coronary Lesions: Serial IVUS-VH and FFR Study. PLoS One. 2016; 11(1): e0147057, doi: 10.1371/journal. pone.0147057, indexed in Pubmed: 26824602. 